\title{
Impact of Initiating Insulin Glargine Disposable Pen Versus Vial/Syringe on Real-World Glycemic Outcomes and Persistence Among Patients with Type 2 Diabetes Mellitus in a Large Managed Care Plan: A Claims Database Analysis
}

\author{
Lin Xie, MA, MS, Steve Zhou, PhD, ${ }^{2}$ Brett W. Pinsky, $\mathrm{MPH}^{3}$ \\ Erin K. Buysman, $\mathrm{MS}^{3}$ and Onur Baser, MS, $\mathrm{PhD}^{1,4}$
}

\begin{abstract}
Background: Diabetes accounts for almost $15 \%$ of all direct healthcare expenditures. Managed care organizations try to reduce costs and improve patient outcomes. Increasing patient persistence with antidiabetes treatment could help achieve these goals.

Subjects and Methods: A retrospective study was conducted using the Optum Research Database (Optum, Eden Prairie, MN) to analyze clinical and economic outcomes associated with initiation of insulin glargine via a disposable pen (GLA-P) or vial and syringe (GLA-V) among adult, insulin-naive patients with type 2 diabetes mellitus (T2DM). Propensity-matched patient cohorts were assessed for persistence with insulin therapy, glycated hemoglobin (A1C), hypoglycemic events (based on diagnosis codes), and healthcare costs (total paid amount of adjudicated claims) after follow-up at 1 year.

Results: In 1,308 matched patients, persistence was significantly higher $(P=0.011)$ and longer $(P=0.001)$ with GLAP. Follow-up A1C values were significantly lower $(P=0.038)$, and decreases in A1C from baseline significantly larger $(P=0.043)$, in GLA-P than in GLA-V. Significantly fewer hypoglycemic events $(P=0.042)$ were experienced, and a lower rate of diabetes-related inpatient admissions $(P=0.008)$ was reported in GLA-P than GLA-V. Despite higher study drug costs with GLA-P than GLA-V, all-cause and diabetes-related healthcare costs were similar.

Conclusions: In insulin-naive patients with T2DM, initiation of insulin glargine using the disposable pen rather than the vial and syringe is associated with higher persistence, better A1C control, and lower rates of hypoglycemia. The higher study drug costs associated with pen use do not increase total all-cause or diabetes-related healthcare costs. This may help treatment selection for patients with T2DM in a managed care setting.
\end{abstract}

\section{Background}

$\mathbf{T}$ He total estimated diabetes-related costs in the United States were $\$ 245$ billion in 2012. ${ }^{1}$ Most of this expenditure relates to hospitalizations and treatment of diabetes complications. ${ }^{2}$ Improving treatment persistence ${ }^{3,4}$ and glycemic control $^{3,5,6}$ and decreasing rates of hypoglycemia ${ }^{7,8}$ have been identified as factors that could result in cost savings in a managed care setting. Other factors that may influence overall cost for managed care organizations are healthcare utilization (hospitalizations, inpatient/outpatient care, specialist vs. primary care), ${ }^{3}$ pharmacy costs, ${ }^{9,10}$ and health services costs (frequency of monitoring, laboratory testing, etc).

Many patients with type 2 diabetes mellitus (T2DM) eventually require injectable insulin therapy. ${ }^{11}$ Long-acting analog basal insulins, such as insulin glargine or detemir, are recommended as initial insulin therapy. ${ }^{11}$ However, conventional administration of these insulins by vial and syringe

\footnotetext{
${ }^{1}$ STATinMED Research, Ann Arbor, Michigan.

${ }^{2}$ Sanofi US Inc., Bridgewater, New Jersey.

${ }^{3}$ Optum, Eden Prairie, Minnesota.

${ }^{4}$ The University of Michigan, Ann Arbor, Michigan.
} 
is associated with significant psychological barriers that can reduce compliance. ${ }^{12,13}$ Medication adherence (or compliance) describes whether a patient is taking medication as prescribed. Treatment persistence refers to the proportion of patients remaining on treatment for a specified period of time. Adherence to medication regimens is generally associated with better health outcomes. ${ }^{14}$ Insulin pen delivery systems seem to improve patient convenience and treatment persistence (and adherence), and thus outcomes, compared with vial and syringe methods of administration. ${ }^{15-17}$ Although adherence to insulin use is a desirable concept, it is hard to measure from healthcare claims data in a reliable way. Therefore, using persistence of insulin treatment is the most feasible approach for retrospective claims studies.

Various studies have investigated clinical parameters and healthcare costs associated with different insulin administration methods for long-, intermediate-, ${ }^{8,18}$ and fast-acting ${ }^{9,10}$ analogs. However, only a few have researched the newer insulin pen devices. ${ }^{15,19-21}$ Furthermore, studies published so far are limited by patient numbers, short duration, and/or the use of combined datasets from multiple insurance providers with large variability in formularies and copay schemes across plans.

The current analysis uses data from a single, large insurance provider to compare patients' real-world persistence with treatment, glycated hemoglobin (A1C) levels, hypoglycemia rates, and healthcare costs.

\section{Subjects and Methods}

\section{Study design and patients}

This retrospective study used medical data, pharmacy data, enrollment information, and laboratory results from the proprietary Optum Research Database utilized by OptumInsight (Optum, Eden Prairie, MN). This database includes claims for pharmacy services (typically submitted electronically by the pharmacy at the time prescriptions are filled) and medical claims or encounter data (which are collected from all available healthcare sites [i.e., inpatient hospital, outpatient hospital, emergency department (ED), physician's office, surgery center, etc.]) for virtually all types of provided services. Data inclusion has been ongoing since 1993. In 2011, there were data available for nearly 13 million individuals with pharmacy and medical benefit coverage.

Data from adult insulin-naive patients, $\geq 18$ years of age, with a diagnosis of T2DM (defined as one or more inpatient stay or two ambulatory visits at least 30 days apart with a primary or secondary diagnosis of International Classification of Diseases, $9^{\text {th }}$ Revision, Clinical Modification [ICD-9$\mathrm{CM}$ ] code $250 . \mathrm{x} 0$ or $250 . \mathrm{x} 2$ ) and who had one or more pharmacy claim for the index treatment-initiation of insulin glargine via a disposable pen (GLA-P) or vial and syringe (GLA-V)—between January 1, 2008 and August 31, 2010. The date of the first claim for insulin glargine was designated as the index date. SoloSTAR ${ }^{\circledR}$ (Sanofi US Inc., Bridgewater, NJ) received Food and Drug Administration approval for use with insulin glargine on May 2, 2007. Patients had to have continuous health plan enrollment for 6 months (180 days) prior to the index date (baseline period) and for 12 months following the index date (follow-up period), one or more claim for an oral antidiabetes drug or glucagon-like peptide-1 receptor agonist during the baseline period, and one or more baseline A1C value. Patients with evidence of pregnancy or gestational diabetes during the baseline or follow-up period were not eligible for inclusion in the analysis.

\section{Study outcomes}

Study outcomes were persistence with index insulin therapy, insulin use, change in $\mathrm{A} 1 \mathrm{C}$ and achievement of $\mathrm{A} 1 \mathrm{C}$ $<7.0 \%$, hypoglycemic events, and healthcare resource utilization and costs during the 12-month follow-up period.

Treatment persistence was defined as the patient remaining on the study drug during the follow-up period without discontinuation or switching after initiation. ${ }^{19}$ Study medication was considered discontinued if the prescription was not refilled within the expected time of medication coverage (the $90^{\text {th }}$ percentile of the time, stratified by the metric quantity supplied, between first and second fills among patients with at least one refill). (For example, among patients who filled a first prescription for a certain medication and had a refill later, $90 \%$ refilled the prescription within a certain period of time $[n$ days]. A patient who had previously filled a first prescription was considered to have discontinued treatment [i.e., did not persist with their treatment] when not refilling within $n$ days.) Patients who restarted their initial medication during follow-up after a period without were considered to be nonpersistent. Sensitivity analyses were also conducted using the $75^{\text {th }}$ and $95^{\text {th }}$ percentiles of the time. Insulin use was measured as the daily average consumption, calculated as the total number of index insulin units dispensed before the last study drug refill divided by the total number of days between initiation and last refill during the follow-up period.

The $\mathrm{A} 1 \mathrm{C}$ was defined as the $\mathrm{A} 1 \mathrm{C}$ value during the 90 days prior to or following the end of the follow-up period. A hypoglycemic event was defined as a healthcare encounter (outpatient, physician's office, ED or other visit, or inpatient admission) with a primary or secondary ICD-9-CM diagnosis code for hypoglycemia or a diagnosis of diabetes with other specified manifestation and no medical claims with ICD-9CM codes for hypoglycemic co-diagnoses. ${ }^{22,23}$ The setting of the hypoglycemic event (outpatient, physician's office, ED, hospital, or other setting) was used as a proxy for severity.

Total all-cause and diabetes-related healthcare resource utilization, healthcare costs, and index study drug costs were assessed. Healthcare utilization was counted as the number of physician's office, outpatient, and ED visits and the number of hospital admissions per patient. Visits with a diagnosis code of 250.xx were considered diabetes-related.

Healthcare costs were calculated as the combined health plan-paid and patient-paid amounts in the follow-up period. Diabetes-related healthcare costs included costs from medical claims with a primary diagnosis of diabetes (ICD-9-CM code 250.xx) or from pharmacy claims for antidiabetes medications (oral antidiabetes drugs, glucagon-like peptide-1, pramlintide, or insulin). Diabetes supply costs, such as glucose meters and test strips, were calculated separately. Costs were adjusted to 2011 dollars to reflect inflation using the medical care component of the U.S. consumer price index. ${ }^{24}$

\section{Measurements and statistical methods}

All study measures were assessed descriptively. To overcome selection bias, GLA-P initiators were matched with GLA-V initiators using 1:1 propensity score matching (PSM). ${ }^{25}$ Variables included in the PSM model were 
determined following review of the prematching descriptive analysis of patient characteristics and other baseline measures and are presented in Table A1 in the Appendix. Patients were only included in the PSM analysis if they had baseline A1C laboratory values available.

Following matching, bivariate comparisons of baseline characteristics and demographics were performed to assess the success of the matching procedure. Independent $t$ tests (for continuous variables) and $\chi^{2}$ tests (for categorical variables) were used based on the distribution of the measure. Bivariate comparisons of economic outcomes were performed using Wilcoxon signed-rank tests. Multivariate modeling was applied to persistence outcomes in the postmatched sample to adjust for potential confounding factors remaining after PSM. Multivariate analyses included Kaplan-Meier analysis, log-rank testing, and Cox proportional hazard models of time to discontinuation $\left(90^{\text {th }}\right.$ percentile).

\section{Results}

\section{Patient selection and baseline characteristics}

Overall, 118,154 patients were identified from the database, and 14,802 unmatched patients were eligible for the final study sample. Of 3,423 patients with baseline A1C data available, 1,308 were evaluated after PSM ( $n=654$ in both GLA-P and GLA-V cohorts). Baseline characteristics were well balanced (Table 1).

\section{Treatment persistence and insulin use}

More GLA-P initiators were persistent with treatment (58.4\% vs. $51.4 \%$ with GLA-V, respectively; $P=0.011$ ) and were persistent for longer (314 days compared with 299 days for GLA-V initiators; $P=0.001)$. The Kaplan-Meier analysis for time to discontinuation indicates that GLA-P initiators

Table 1. Baseline Characteristics for Initiators of Insulin Glargine by Disposable Pen or by Vial and Syringe Following Propensity Score Matching

\begin{tabular}{|c|c|c|c|}
\hline Characteristic & GLA-P initiators $(\mathrm{n}=654)$ & GLA-V initiators $(\mathrm{n}=654)$ & $\mathrm{P}$ value \\
\hline \multicolumn{4}{|l|}{ Age range (years) $[n(\%)]$} \\
\hline $18-39$ & $45(6.9)$ & $44(6.7)$ & 0.913 \\
\hline $40-64$ & $551(84.3)$ & $565(86.4)$ & 0.274 \\
\hline $65-74$ & $54(8.3)$ & $42(6.4)$ & 0.203 \\
\hline$\geq 75$ & $4(0.6)$ & $3(0.5)$ & 0.705 \\
\hline Female $[n(\%)]$ & $279(42.7)$ & $288(44.0)$ & 0.616 \\
\hline \multicolumn{4}{|l|}{ Plan type $[n(\%)]$} \\
\hline Health maintenance organization & $97(14.8)$ & $118(18.0)$ & 0.117 \\
\hline Point-of-service & $354(54.1)$ & $323(49.4)$ & 0.086 \\
\hline Preferred provider organization & $84(12.8)$ & $82(12.5)$ & 0.868 \\
\hline Other & $119(18.2)$ & $131(20.0)$ & 0.399 \\
\hline Modified CCI [mean (SD)] & $1.15(1.8)$ & $1.09(1.6)$ & 0.486 \\
\hline Any hypoglycemia $[n(\%)]$ & $20(3.1)$ & $24(3.7)$ & 0.540 \\
\hline \multicolumn{4}{|l|}{ Antidiabetes therapy $[n(\%)]$} \\
\hline Metformin & $392(59.9)$ & $409(62.5)$ & 0.335 \\
\hline Sulfonylureas & $401(61.3)$ & $417(63.8)$ & 0.361 \\
\hline DPP-4 inhibitor & $155(23.7)$ & $162(24.8)$ & 0.652 \\
\hline GLP-1 receptor analog & $95(14.5)$ & $90(13.8)$ & 0.692 \\
\hline Thiazolidinedione & $240(36.7)$ & $226(34.6)$ & 0.419 \\
\hline Pramlintide & $1(0.2)$ & $1(0.2)$ & 1.000 \\
\hline$\alpha$-Glucosidase inhibitor & $5(0.8)$ & $4(0.6)$ & 0.738 \\
\hline OAD MPR [mean (SD)] & $0.69(0.3)$ & $0.70(0.3)$ & 0.355 \\
\hline $\mathrm{A} 1 \mathrm{C}(\%)[$ mean $(\mathrm{SD})]$ & $9.41(2.1)$ & $9.48(2.2)$ & 0.569 \\
\hline \multicolumn{4}{|l|}{ A1C range $[n(\%)]$} \\
\hline$<7.0 \%$ & $76(11.6)$ & $65(9.9)$ & 0.327 \\
\hline$\geq 7.0-8.0 \%$ & $107(16.4)$ & $107(16.4)$ & 1.000 \\
\hline$\geq 8.0-9.0 \%$ & $122(18.7)$ & $122(18.7)$ & 1.000 \\
\hline$\geq 9.0$ & $349(53.4)$ & $360(55.0)$ & 0.542 \\
\hline \multicolumn{4}{|l|}{ All-cause healthcare utilization $[n(\%)]$} \\
\hline ED visit & $56(8.6)$ & $57(8.7)$ & 0.922 \\
\hline Inpatient admission & $93(14.2)$ & $94(14.4)$ & 0.937 \\
\hline \multicolumn{4}{|c|}{ Diabetes-related healthcare utilization $[n(\%)]$} \\
\hline ED visit & $21(3.2)$ & $17(2.6)$ & 0.510 \\
\hline Inpatient admission & $68(10.4)$ & $68(10.4)$ & 1.000 \\
\hline \multicolumn{4}{|c|}{ Total healthcare costs (U.S. dollars) [mean (SD)] } \\
\hline All-cause & $10,442(25,611)$ & $10,069(23,567)$ & 0.784 \\
\hline Diabetes-related & $3,739(9,341)$ & $3,662(9,933)$ & 0.885 \\
\hline
\end{tabular}

A1C, glycated hemoglobin; CCI, Charlson Comorbidity Index; DPP-4, dipeptidyl peptidase-4; ED, emergency department; GLA-P, insulin glargine pen; GLA-V, insulin glargine vial and syringe; GLP-1, glucagon-like peptide-1; MPR, medication possession ratio; OAD, oral antidiabetes drug; PSM, propensity score matching. 


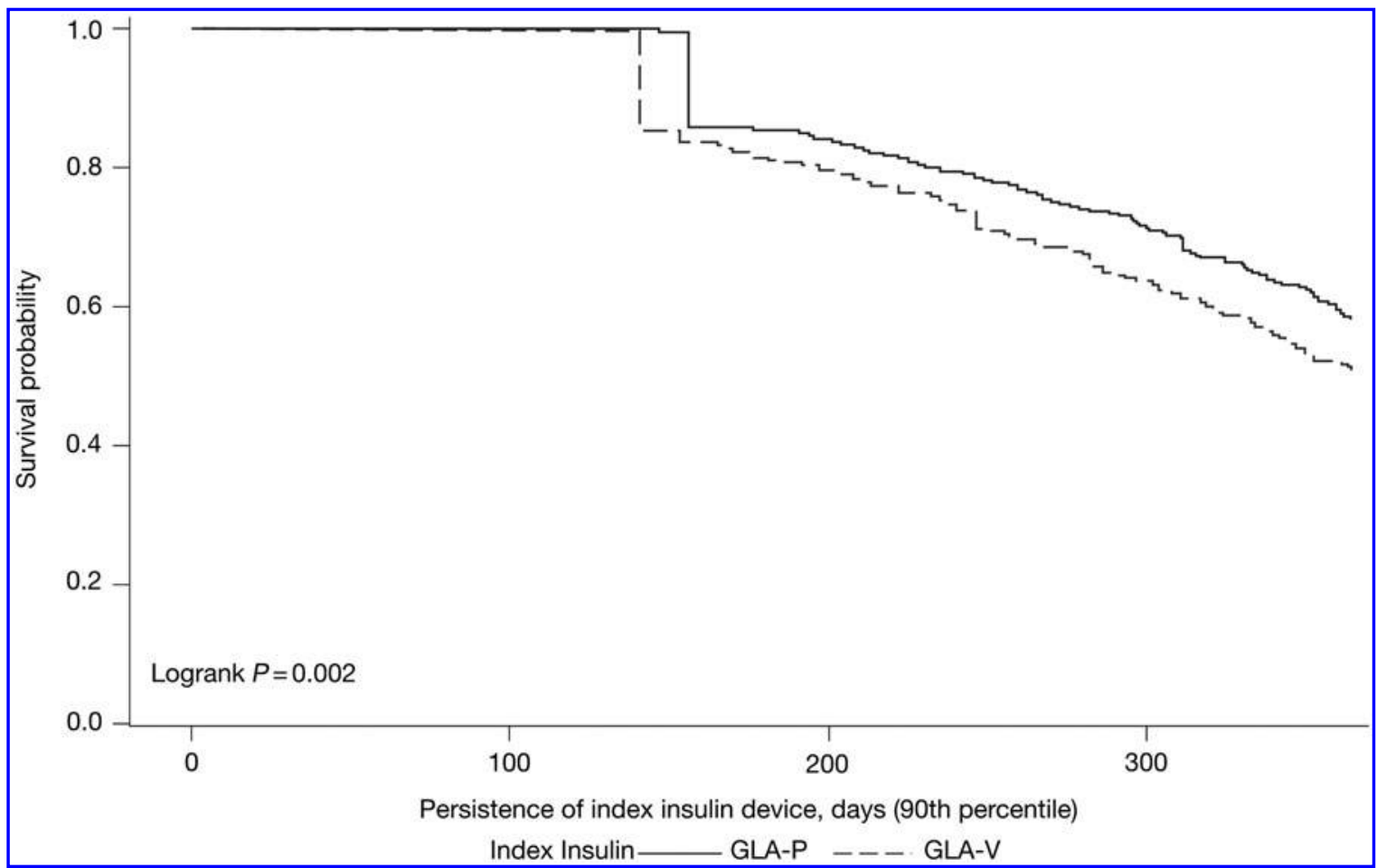

FIG. 1. Kaplan-Meier curve and log-rank test for time to treatment discontinuation ( $90^{\text {th }}$ percentile) for matched initiators of insulin glargine by disposable pen (GLA-P) (solid line) or vial and syringe (GLA-V) (dashed line).

persisted with treatment significantly longer than GLA-V initiators $(P=0.002$, log-rank test) (Fig. 1). Cox proportional hazards modeling for time to treatment discontinuation demonstrated a slower rate of treatment discontinuation among GLA-P initiators (hazard ratio $=0.78, P=0.003$ ).

Insulin use among the GLA-P and the GLA-V initiators was not significantly different (mean daily average consumption, 24.3 units/day with GLA-P and 23.8 units/day with GLA-V; $P=0.750)$.

\section{Glycemic control}

The A1C change from baseline was assessed in a subgroup of 996 matched patients with both baseline and follow-up A1C data available ( $n=498$ in each). In this subset of patients, mean baseline A1C was $9.5 \%$ for GLA-P initiators and $9.4 \%$ for
GLA-V initiators $(P=0.816)$. GLA-P initiators had significantly lower average follow-up A1C values compared with GLA-V initiators $(8.3 \%$ vs. $8.5 \% ; P=0.038)$. GLA-P initiators also experienced a larger decrease in $\mathrm{A} 1 \mathrm{C}$ from baseline compared with GLA-V initiators $(-1.2 \%$ vs. $-0.9 \% ; P=0.043)$. The proportion of patients achieving an $\mathrm{A} 1 \mathrm{C}<7 \%$ was similar in both cohorts $(26.9 \%$ vs. $23.3 \% ; P=0.188)$ (Table 2$)$.

\section{Hypoglycemia}

Medical claims showed that patients initiating GLA-P were significantly less likely to experience hypoglycemia requiring physician care than those initiating GLA-V: $5.1 \%$ of GLA-P initiators and $7.8 \%$ of GLA-V $(P=0.042)$ (Fig. 2). GLA-P initiators also had lower rates of outpatient, ED, and inpatient/ED-related hypoglycemic events (Fig. 2A). GLA-P

Table 2. 12-Month Follow-Up Glycated Hemoglobin (A1C) Parameters Among Matched Initiators of Insulin Glargine by Disposable Pen or by Vial and Syringe with Baseline and Follow-Up A1C Data Available

\begin{tabular}{|c|c|c|c|}
\hline AlC parameter & $G L A-P$ initiators $(\mathrm{n}=498)$ & $G L A-V$ initiators $(\mathrm{n}=498)$ & $\mathrm{P}$ value \\
\hline Baseline A1C (\%) [mean (SD)] & $9.5(2.2)$ & $9.4(2.1)$ & 0.816 \\
\hline Follow-up A1C (\%) [mean (SD)] & $8.3(1.9)$ & $8.5(1.9)$ & 0.038 \\
\hline \multicolumn{4}{|l|}{ Follow-up A1C range $[n(\%)]$} \\
\hline$<7.0 \%$ & $134(26.9)$ & $116(23.3)$ & 0.188 \\
\hline$\geq 7.0-8.0 \%$ & 134 (26.9) & 117 (23.5) & 0.215 \\
\hline$\geq 8.0-9.0 \%$ & $79(15.9)$ & $96(19.3)$ & 0.157 \\
\hline$\geq 9.0 \%$ & $151(30.3)$ & $169(33.9)$ & 0.222 \\
\hline Change $(\%)$ in $\mathrm{A} 1 \mathrm{C}$ from baseline [mean (SD)] & $-1.2(2.2)$ & $-0.9(2.2)$ & 0.043 \\
\hline
\end{tabular}

A1C, glycated hemoglobin; GLA-P, insulin glargine pen; GLA-V, insulin glargine vial and syringe. 

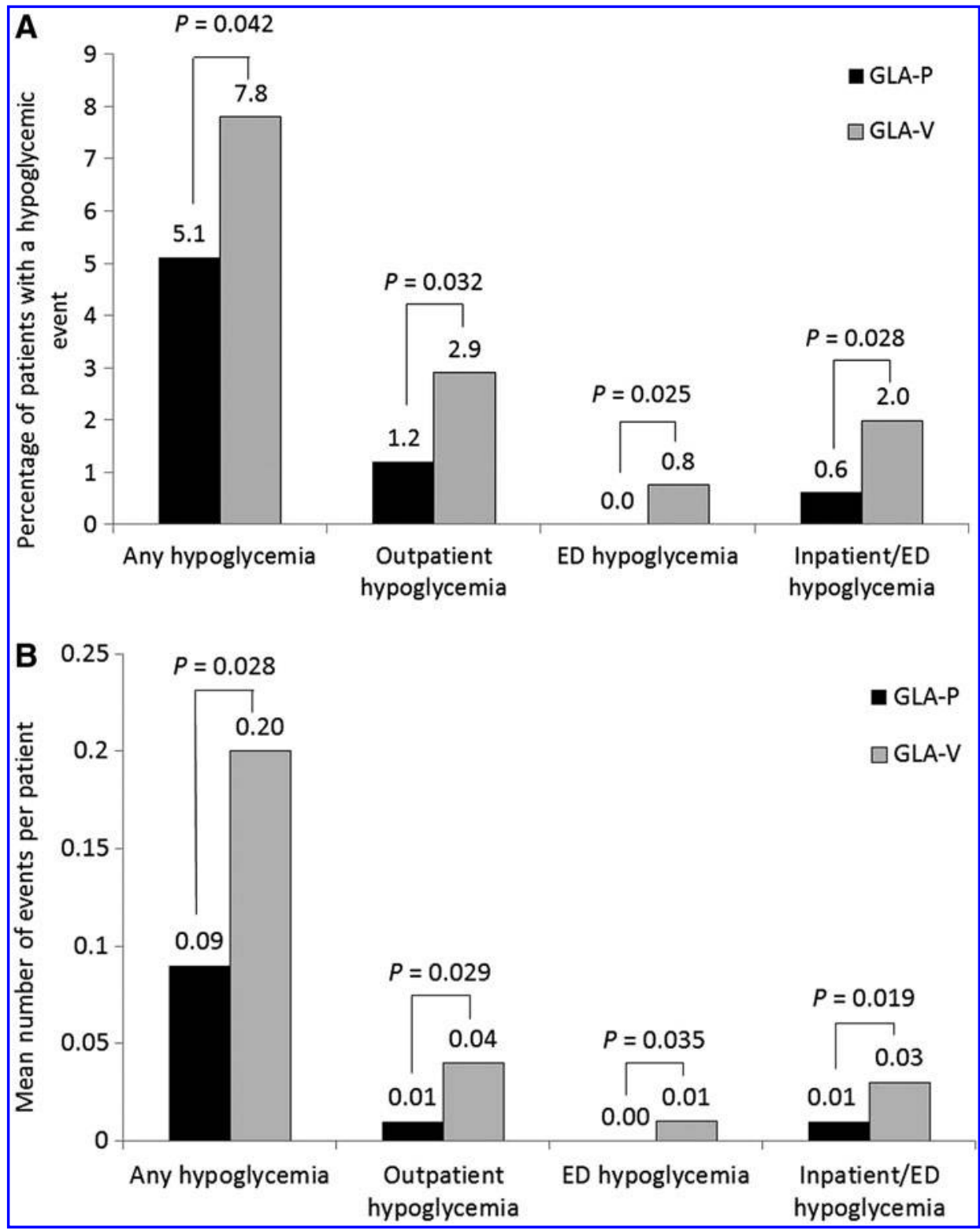

FIG. 2. Follow-up hypoglycemia among matched initiators of insulin glargine by disposable pen (GLA-P) or vial and syringe (GLA-V): (A) rate of hypoglycemic events and (B) mean number of events per patient. ED, emergency department.

initiators had significantly fewer overall mean number of hypoglycemic events per patient (0.09 vs. $0.20 ; P=0.028)$, outpatient events ( 0.01 vs. $0.04 ; P=0.029)$, ED events $(0.00$ vs. $0.01 ; P=0.035)$, and inpatient/ED events (0.01 vs. 0.03 ; $P=0.019$ ) compared with GLA-V initiators (Fig. 2B).

\section{Healthcare utilization and costs}

During the 1-year follow-up period, the rate of diabetesrelated inpatient admissions was significantly lower among GLA-P initiators compared with GLA-V initiators (9.6\% vs. $14.4 \% ; P=0.008)$. There were no significant differences in all-cause or diabetes-related physician office visits, outpatient visits, or ED visits (Fig. 3).

GLA-P initiators had higher study drug costs than GLA-V initiators ( $\$ 1,141$ vs. $\$ 927 ; P<0.001)$. However, this did not translate to higher diabetes-related costs as both total all- cause and total diabetes-related healthcare costs were similar; mean total all-cause costs were $\$ 21,451$ versus $\$ 21,043$ $(P=0.711)$, and mean total diabetes-related costs were $\$ 7,528$ versus $\$ 7,971(P=0.772)$ for GLA-P and GLA-V initiators, respectively (Table 3 ).

\section{Discussion}

This retrospective real-world analysis of information from a database associated with a single, large insurance provider compared clinical and economic outcomes among insulinnaive patients with T2DM who initiated insulin glargine using disposable pen or vial and syringe. After follow-up at 1 year, patients initiating insulin glargine using the disposable pen had higher persistence rates (58.4\% vs. 51.4\%), a larger decrease in $\mathrm{A} 1 \mathrm{C}$ from baseline, and significantly lower rates of hypoglycemic events compared with those using vial and 


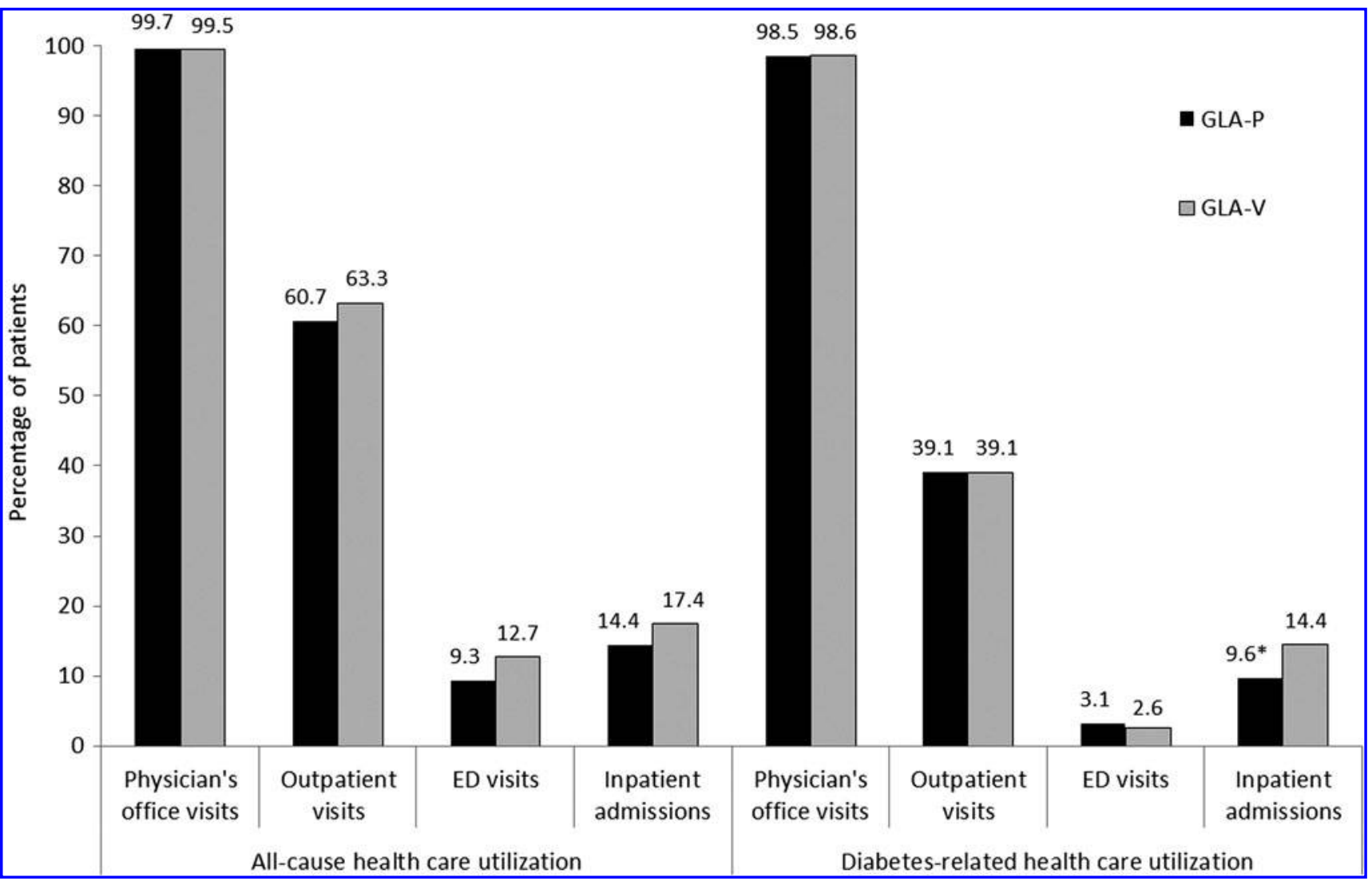

FIG. 3. Follow-up healthcare resource utilization among matched initiators of insulin glargine by disposable pen (GLA-P) or vial and syringe (GLA-V). ${ }^{*} P=0.008$ versus GLA-V. ED, emergency department.

syringe. Previous studies have shown that patients find the disposable pen easy to use, allowing easy dose selection and readout, which leads to better dosing accuracy. ${ }^{17,26}$ Improved dosing accuracy could explain the better clinical outcomes observed in patients initiating insulin glargine using the disposable pen. The ease of use and better dosing accuracy of the pen device can improve medication use behavior, including treatment persistence, thereby potentially contributing to improved clinical outcomes. ${ }^{27,28}$ Initiation of insulin glargine therapy by disposable pen also led to a significant

Table 3. 12-Month Follow-Up Healthcare Costs Among Propensity Score-Matched Initiators of Insulin Glargine by Disposable Pen or by Vial and Syringe

\begin{tabular}{lccc}
\hline & \multicolumn{2}{c}{ U.S. dollars [mean $(S D)]$} & \\
\cline { 2 - 4 } Follow-up healthcare costs & GLA-P initiators $(\mathrm{n}=654)$ & GLA-V initiators $(\mathrm{n}=654)$ & P value \\
\hline Total all-cause costs & $21,451(40,609)$ & $21,043(41,648)$ & 0.711 \\
Pharmacy costs & $6,118(6,254)$ & $5,671(5,407)$ & 0.055 \\
Physician office costs & $2,892(7,736)$ & $5,597(8,873)$ & 0.263 \\
Outpatient costs & $4,496(11,657)$ & $104(756)$ & 0.860 \\
ED costs & $44(320)$ & $4,092(17,214)$ & 0.009 \\
Inpatient costs & $4,344(20,765)$ & $3,156(13,912)$ & 0.417 \\
Other costs & $3,556(15,818)$ & $7,971(16,409)$ & 0.719 \\
Total diabetes-related costs & $7,528(14,281)$ & $2,232(1,645)$ & 0.772 \\
Pharmacy costs & $2,554(1,748)$ & $666(727)$ & $<0.001$ \\
Physician office costs & $718(688)$ & $1,849(8,991)$ & 0.042 \\
Outpatient costs & $1,554(6,567)$ & $14(151)$ & 0.878 \\
ED costs & $7(95)$ & $2,009(7,868)$ & 0.620 \\
Inpatient costs & $1,498(7,822)$ & $1,200(6,925)$ & 0.043 \\
Other costs & $1,196(6,780)$ & $927(773)$ & 0.623 \\
Study drug costs & $1,141(906)$ & $373(460)$ & $<0.001$ \\
Diabetes supply costs & $423(501)$ & 0.046
\end{tabular}

A1C, glycated hemoglobin; ED, emergency department; GLA-P, insulin glargine pen; GLA-V, insulin glargine vial and syringe; PSM, propensity score matching. 
reduction in the rate of diabetes-related inpatient admission during follow-up. Although pharmacy costs were higher for the disposable pen $(5.3 \%$ vs. $4.4 \%$ of total healthcare costs, respectively), total all-cause and diabetes-related healthcare costs were similar. Other studies of managed care databases have reported similar results among patients administering insulin glargine via disposable pen: pen use was associated with higher medication costs compared with vial and syringe, whereas total healthcare resource utilization and costs were similar between the two administration methods. ${ }^{19,29}$ The authors of these studies have suggested that this may be due to improved treatment persistence, lower rates of hypoglycemia, and fewer hospitalizations in patients using the insulin glargine disposable pen. ${ }^{19,29}$ Thus, initiation of insulin glargine using the disposable pen rather than the vial and syringe increases patient persistence and improves clinical outcomes. The additional pharmacy costs for the disposable pen are offset by lower costs related to inpatient utilization. Therefore, the pen use has no implications for expenditure by the health insurer.

Like many other real-world claims data analyses, studies assessing the influence of copayment on treatment persistence are carried out on large, multiprovider/multiplan datasets. The large numbers of patients included in such datasets add to the robustness of the observed outcomes and to the generalizability to the U.S. population as a whole. However, it is unclear whether the outcomes of those studies are replicable in other, more specific settings. For example, multiprovider/multiplan datasets may combine data from providers/plans with different copay rates (and possibly with some variation in the formularies) and generally do not allow for the observation of concurrently implemented interventions that may have an effect on treatment persistence and healthcare resource utilization.

In the current study, copayment was included as a variable in the PSM model. The majority of patients in each treatment cohort had an average copayment of more than $\$ 31$ for the index insulin. However, copayment may lead to treatment switching, treatment interruptions, or patients taking the drug less frequently than prescribed to extend its period of use. ${ }^{30} \mathrm{In}$ a study of oral antidiabetes drug treatment, increased copayment was a significant predictor of treatment failure. ${ }^{31}$ Therefore, increased healthcare cost sharing between the payer and the patient, by means of higher copayments for the patient, leads to a decreased treatment persistence/adherence and may have deleterious effects on patients' health. Conversely, lower copayment (\$0-9) has been reported to result in significantly higher adherence to oral antidiabetes drugs, lower total healthcare expenditure, and lower risk of hospitalization compared with patients with a higher copayment $(\geq \$ 20) .^{32}$ Therefore, it is paramount that cost containment approaches are value-based, to ensure both improved clinical outcomes and cost containment in the long term. ${ }^{33}$ To confirm that copayment did not influence outcomes, we removed this variable from the PSM model and re-evaluated the results. Following the removal of copayment, overall results were similar to those obtained with the match on copayment (data not shown).

A strength of our study is that it is based on data from a single large insurance provider to maximize the robustness of the results. This approach allowed for subanalyses of issues of particular importance to decision-makers in the managed care community (such as the role of copays) and increased the generalizability of the conclusions to plans of a similar nature.

This study has several limitations, which are described in detail in the Supplementary Data (Supplementary Data are available online at www.liebertonline.com/dia). In brief, the use of administrative claims databases is associated with inherent and well-known limitations, including deficient information on dosage, duration of disease, and body weight. Furthermore, retrospective analyses of claims databases may introduce a risk of selection bias and confounding and do not allow assessment of site-specific shortages or stocking issues. Our study is based on claims data from a managed care population and may not be fully generalizable to all T2DM patients. Finally, the time frame of our study may have been too short to detect changes in the risk of complications that heavily impact costs in the longer term.

\section{Conclusions}

This real-world database study of insulin-naive patients with T2DM shows that initiation of insulin glargine treatment administered with a disposable pen improved patients' clinical outcomes without additional healthcare expenditure when compared with insulin glargine initiation using vial and syringe. Specifically, the disposable pen was associated with higher treatment persistence, better glycemic control, and lower rates of hypoglycemia but similar total all-cause or diabetes-related healthcare costs. This information may help the selection of the most appropriate treatment modality in a managed care setting.

\section{Acknowledgments}

This study was funded by Sanofi US Inc. The authors gratefully acknowledge the support provided by Juan Du of STATinMED Research. The authors received writing/ editorial support in the preparation of this manuscript provided by Tessa Hartog, PhD, of Excerpta Medica, funded by Sanofi US Inc.

\section{Author Disclosure Statement}

L.X. and O.B. are employees of STATinMED Research, under contract with Sanofi US Inc. S.Z. is an employee of Sanofi US Inc. B.P. was an employee of Optum at the time of this study, and E.B. is a current employee of Optum, under contract with Sanofi US Inc.

L.X. contributed to the development of the study concept, conducted statistical analyses, and co-wrote the study report. S.Z. proposed and co-developed the study concept, codeveloped the analysis plan, and interpreted the study data. B.P. is a senior researcher who contributed to the development of the study concept, co-wrote the study report, co-designed the study, and assisted in collecting, analyzing, and interpreting the study data. E.B. is a senior researcher who contributed to the development of the study concept, co-designed the study, and assisted in collecting, analyzing, and interpreting the study data. O.B. co-developed the statistical analysis plan, provided input for the study report, advised on data cleaning, and assisted in interpreting the data. All authors have provided input during manuscript development, have reviewed the manuscript, and have approved the final version prior to submission. 


\section{References}

1. American Diabetes Association: Economic costs of diabetes in the U.S. in 2012. Diabetes Care 2013;36:1033-1046.

2. Selby JV, Ray GT, Zhang D, Colby CJ: Excess costs of medical care for patients with diabetes in a managed care population. Diabetes Care 1997;20:1396-1402.

3. Stephens JM, Botteman MF, Hay JW: Economic impact of antidiabetic medications and glycemic control on managed care organizations: a review of the literature. J Manag Care Pharm 2006;12:130-142.

4. Balkrishnan R, Rajagopalan R, Camacho FT, Huston SA, Murray FT, Anderson RT: Predictors of medication adherence and associated health care costs in an older population with type 2 diabetes mellitus: a longitudinal cohort study. Clin Ther 2003;25:2958-2971.

5. Wagner EH, Sandhu N, Newton KM, McCulloch DK, Ramsey SD, Grothaus LC: Effect of improved glycemic control on health care costs and utilization. JAMA 2001;285: 182-189.

6. Shetty S, Secnik K, Oglesby AK: Relationship of glycemic control to total diabetes-related costs for managed care health plan members with type 2 diabetes. J Manag Care Pharm 2005;11:559-564.

7. Heaton A, Martin S, Brelje T: The economic effect of hypoglycemia in a health plan. Manag Care Interface 2003; 16:23-27.

8. Bullano MF, Al-Zakwani IS, Fisher MD, Menditto L, Willey VJ: Differences in hypoglycemia event rates and associated cost-consequence in patients initiated on longacting and intermediate-acting insulin products. $\underline{\text { Curr Med }}$ Res Opin 2005;21:291-298.

9. Chen K, Chang EY, Summers KH, Obenchain RL, YuIsenberg KS, Sun P: Comparison of costs and utilization between users of insulin lispro versus users of regular insulin in a managed care setting. J Manag Care Pharm 2005; 11:376-382.

10. Hall JA, Summers KH, Obenchain RL: Cost and utilization comparisons among propensity score-matched insulin lispro and regular insulin users. J Manag Care Pharm 2003; 9:263-268.

11. Inzucchi SE, Nauck M, Bergenstal RM, Peters AL, Buse JB, Tsapas A, Diamant M, Wender R, Ferrannini E, Matthews DR: Management of hyperglycemia in type 2 diabetes: a patient-centered approach. Diabetes Care 2012;35: 1364-1379.

12. Karter AJ, Subramanian U, Saha C, Crosson JC, Parker MM, Swain BE, Moffet HH, Marrero DG: Barriers to insulin initiation: the Translating Research Into Action for Diabetes Insulin Starts Project. Diabetes Care 2010;33: 733-735.

13. Korytkowski M, Niskanen L, Asakura T: FlexPen: addressing issues of confidence and convenience in insulin delivery. Clin Ther 2005;27(Suppl B):S89-S100.

14. DiMatteo MR, Giordani PJ, Lepper HS, Croghan TW: Patient adherence and medical treatment outcomes: a metaanalysis. Med Care 2002;40:794-811.

15. Xie L, Zhou S, Wei W, Gill J, Pan C, Baser O: Does pen help? A real-world outcomes study of switching from vial to disposable pen among insulin glargine-treated patients with type 2 diabetes mellitus. Diabetes Technol Ther 2013; 15:230-236.

16. Buysman E, Conner C, Aagren M, Bouchard J, Liu F: Adherence and persistence to a regimen of basal insulin in a pre-filled pen compared to vial/syringe in insulin-naïve patients with type 2 diabetes. Curr Med Res Opin 2011;27: 1709-1717.

17. Anderson BJ, Redondo MJ: What can we learn from patient-reported outcomes of insulin pen devices? J Diabetes Sci Technol 2011;5:1563-1571.

18. Borah BJ, Darkow T, Bouchard J, Aagren M, Forma F, Alemayehu B: A comparison of insulin use, glycemic control, and health care costs with insulin detemir and insulin glargine in insulin-naive patients with type 2 diabetes. Clin Ther 2009;31:623-631.

19. Davis SN, Wei W, Garg S. Clinical impact of initiating insulin glargine therapy with disposable pen versus vial in patients with type 2 diabetes mellitus in a managed care setting. Endocr Pract 2011;17:845-852.

20. Lee WC, Balu S, Cobden D, Joshi AV, Pashos CL: Medication adherence and the associated health-economic impact among patients with type 2 diabetes mellitus converting to insulin pen therapy: an analysis of third-party managed care claims data. Clin Ther 2006;28:1712-1725.

21. Pawaskar MD, Camacho FT, Anderson RT, Cobden D, Joshi AV, Balkrishnan R: Health care costs and medication adherence associated with initiation of insulin pen therapy in Medicaid-enrolled patients with type 2 diabetes: a retrospective database analysis. Clin Ther 2007;29:1294-1305.

22. Ginde AA, Blanc PG, Lieberman RM, Camargo CA Jr: Validation of ICD-9-CM coding algorithm for improved identification of hypoglycemia visits. BMC Endocr Disord 2008;8:4.

23. Zhao Y, Campbell CR, Fonseca V, Shi L: Impact of hypoglycemia associated with antihyperglycemic medications on vascular risks in veterans with type 2 diabetes. Diabetes Care 2012;35:1126-1132.

24. U.S. Department of Labor, Bureau of Labor Statistics. Consumer Price Index. Chained Consumer Price Index for all urban consumers (C-CPI-U) 1999-2008, Medical Care. Series ID: SUUR0000SAM. 2008. http://data.bls.gov/ cgi-bin/surveymost?su (accessed January 28, 2011).

25. Rosenbaum PR, Rubin DB: The central role of the propensity score in observational studies for causal effects. Biometrika 1983;70:41-55.

26. Hancu N, Czupryniak L, Genestin E, Sourij H: A PanEuropean and Canadian prospective survey to evaluate patient satisfaction with the SoloSTAR insulin injection device in type 1 and type 2 diabetes. J Diabetes Sci Technol 2011;5:1224-1234.

27. Pladevall M, Williams LK, Potts LA, Divine G, Xi H, Lafata JE: Clinical outcomes and adherence to medications measured by claims data in patients with diabetes. Diabetes Care 2004;27:2800-2805.

28. Wei W, Pan C, Xie L, Baser O: Real-world insulin treatment persistence among patients with type 2 diabetes: measures, predictors and outcomes. Endocr Pract 2014;20:52-61.

29. Lin J, Ling J, Wei W, Pan C: Outcomes of initiating insulin glargine therapy with disposable pen or vial among patients with type 2 diabetes in a U.S. managed care health plan. J Diabetes Sci Technol 2012;6:A90-A91.

30. Saito EP, Davis JW, Harrigan RC, Juarez D, Mau MK. Copayment level and drug switching: findings for type 2 diabetes. Am J Pharm Benefits 2010;2:412-420.

31. Barron J, Wahl P, Fisher M, Plauschinat C: Effect of prescription copayments on adherence and treatment failure with oral antidiabetic medications. Pharm Ther 2008;33:532-541. 
32. Colombi AM, Yu-Isenberg K, Priest J: The effects of health plan copayments on adherence to oral diabetes medication and health resource utilization. J Occup Environ Med 2008; 50:535-541.

33. Fendrick AM, Chernew ME: Value-based insurance design: aligning incentives to bridge the divide between quality improvement and cost containment. Am J Manag Care 2006;12(Special Issue):SP5-SP10.

\section{Appendix}

Table A1. Baseline Covariates Controlled For During Propensity Score Matching

\section{Age \\ Female \\ Plan type \\ HMO \\ POS \\ PPO}

Geographic region

Northeast

Midwest

South

West

Copay for index insulin

\$16-30

$\$ 31+$

Baseline comorbidity

Modified Charlson Comorbidity Indexes

Myocardial infarction

Congestive heart failure

Cerebrovascular disease

Dementia

Paraplegia and hemiplegia

Renal disease

Cancer

Hypertension

Hyperlipidemia

Obesity

Severe mental illness

Other ischemic heart disease and angina

Amputation and ulceration

Ocular diseases that lead to visual impairment Dyslipidemia

Baseline hypoglycemia Any hypoglycemia Inpatient/ER hypoglycemia

Baseline diabetes-related medication use Sulfonylureas

DPP-4

GLP-1
Address correspondence to:

Steve Zhou, PhD

Sanofi US, Inc.

55 Corporate Drive

Bridgewater, NJ 08807-5925

E-mail: steve.zhou@sanofi.com
TABle A1. (Continued)

Baseline concomitant medication use

Statins

ACE

ARB

$\alpha$-Blocker

Calcium channel blocker

Adrenergic receptor agonists

Aldosterone antagonists

Baseline OAD MPR

Baseline all-cause healthcare utilization

Inpatient admissions

30-day hospitalization

Baseline diabetes-related healthcare utilization Inpatient admissions 30-day hospitalization

Baseline all-cause healthcare costs

Total costs

Baseline diabetes-related healthcare costs

Total costs

ED costs

Baseline medication dispensings

Baseline A1c

$<7 \%$

$\geq 7-8 \%$

$\geq 8-9 \%$

Baseline microalbuminuria $3.5-35 \mathrm{~g} / \mathrm{mol}$ for female $2.5-25 \mathrm{~g} / \mathrm{mol}$ for male

Baseline serum creatinine value (flagged between 0.5 and $1.4 \mathrm{mg} / \mathrm{dL}$ )

$\mathrm{ACE}$, angiotensin converting enzyme; ARB, angiotensin II receptor blocker; DPP-4, dipeptidyl peptidase-4; ED, emergency department; ER, emergency room; GLP-1, glucagon-like peptide-1; HMO, health maintenance organization; MPR, medication possession ratio; OAD, oral antidiabetes drug; POS, point of service; PPO, preferred provider organization. 


\section{This article has been cited by:}

1. Gudiksen Niels, Hofstätter Thibaud, Rønn Birgitte B., Sparre Thomas. 2017. FlexTouch: An Insulin Pen-Injector with a Low Activation Force Across Different Insulin Formulations, Needle Technologies, and Temperature Conditions. Diabetes Technology \&. Therapeutics 19:10, 603-607. [Abstract] [Full Text HTML] [Full Text PDF] [Full Text PDF with Links]

2. Paolo Pozzilli, Paul Norwood, Esteban J?dar, Melanie J. Davies, Tibor Ivanyi, Honghua Jiang, D. Bradley Woodward, Zvonko Milicevic. 2017. Placebo-controlled, randomized trial of the addition of once-weekly glucagon-like peptide-1 receptor agonist dulaglutide to titrated daily insulin glargine in patients with type 2 diabetes (AWARD-9). Diabetes, Obesity and Metabolism 19:7, 1024-1031. [Crossref]

3. Wenhui Wei, Jenny Jiang, Youbei Lou, Sohini Ganguli, Mark S. Matusik. 2017. Benchmarking Insulin Treatment Persistence Among Patients with Type 2 Diabetes Across Different U.S. Payer Segments. Journal of Managed Care \& Specialty Pharmacy 23:3, 278-290. [Crossref]

4. Abdulaziz Bin rsheed, Ian Chenoweth. 2017. Barriers that practitioners face when initiating insulin therapy in general practice settings and how they can be overcome. World Journal of Diabetes 8:1, 28. [Crossref]

5. Samuel Stolpe, Michel A. Kroes, Neil Webb, Tami Wisniewski. 2016. A Systematic Review of Insulin Adherence Measures in Patients with Diabetes. Journal of Managed Care \& Specialty Pharmacy 22:11, 1224-1246. [Crossref]

6. Ronan Roussel, Bernard Charbonnel, Mourad Behar, Julie Gourmelen, Corinne Emery, Bruno Detournay. 2016. Persistence with Insulin Therapy in Patients with Type 2 Diabetes in France: An Insurance Claims Study. Diabetes Therapy 7:3, 537-549. [Crossref]

7. Pieralessandro Lasalvia, Julián Esteban Barahona-Correa, Diana Marcela Romero-Alvernia, Sebastián Gil-Tamayo, Camilo Castañeda-Cardona, Juan Gabriel Bayona, Juan José Triana, Andrés Felipe Laserna, Miguel Mejía-Torres, Paula RestrepoJimenez, Juliana Jimenez-Zapata, Diego Rosselli. 2016. Pen Devices for Insulin Self-Administration Compared With Needle and Vial. Journal of Diabetes Science and Technology 10:4, 959-966. [Crossref]

8. Sarah Thayer, Wing Chow, Stephanie Korrer, Richard Aguilar. 2016. Real-world evaluation of glycemic control among patients with type 2 diabetes mellitus treated with canagliflozin versus dipeptidyl peptidase- 4 inhibitors. Current Medical Research and Opinion 32:6, 1087-1096. [Crossref]

9. Alice P. S. Kong, Juliana C. N. Chan. 2015. Hypoglycemia and Comorbidities in Type 2 Diabetes. Current Diabetes Reports 15:10. . [Crossref] 